\title{
The Role of Current Distribution in Cathodic Protection
}

\author{
Homer D. Holler
}

\begin{abstract}
The paper outlines a procedure for determining current distribution over an electrode surface, as required in eathodic protection or in electroplating when the electrode potential bears a known relation to current density; and shows the relation of current distribution to resistance of current path and counter electromotive force. The primary current distribution (without polarization) is also computed. A method is suggested for determining the electrode potential-current density relation over an extensive surface in a uniform medium. In a nonuniform medium, the determination of current density by measurement of electrode potential becomes complex. In such a case the potential criterion of cathodic protection must be relied upon.
\end{abstract}

\section{Introduction}

The electrical requirement for complete cathodic protection of a metal from corrosion was demonstrated years ago by Mears and Brown [1]. ${ }^{1}$ Their work established a criterion based on the equalization of surface potentials, which is accomplished by polarizing the cathodic areas of the metal until their potentials become equal to the "open-circuit" potential of the anodic areas. As a result, the current leaving the anodic areas, and consequently the equivalent rate of corrosion, is reduced to zero. The mechanism of the process is based on increasing the polarization of the cathodic areas by the application of external current to those areas.

Let us consider the potential ${ }^{2}$ relations, when a voltage, $E$, is applied to a galvanic couple in which the potential of the cathodic area is $e_{c}$ and that of the anodic area is $e_{a}$. If $E$ is gradually increased from zero, current $I$ will flow first to the cathodic area when $E>E_{G}$, where $E_{G}$ is the potential of the couple and lies between $e_{a}$ and $e_{c}$. That is,

$$
\begin{aligned}
E_{G} & =e_{a}-i_{0} r_{a} \\
& =e_{c}+i_{0} r_{c},
\end{aligned}
$$

where

$i_{0}=$ the current circulating within the couple before $E$ is applied

$r_{a}=$ resistance of the anodic path

$r_{c}=$ resistance of the cathodic path.

Only when $E>e_{a}$ does current begin flowing to the initially anodic area, and this occurs when $e_{c}+I_{c} r_{c}=e_{a}$, where $I_{c}$ is the total cathodic current; and $e_{a}$ becomes equal to $E_{a}$ the open-circuit potential.

As the potential relations within a galvanic couple are such that applied current flows to the cathodic areas as required, it may at first appear that there is no problem of current distribution in the application of cathodic protection. That is, the problem seems

1 Figures in brackets indicate the literature references at the end of this paper. 2 The term "potential" used herein really means a potential difference; and if a current is flowing, a polarized potential is understood. to be automatically solved. However, let us consider the typical condition when galvanic corrosion is most insidious, that is, localized pitting. Then, the anodic area is negligible, and the problem becomes a practical one of obtaining current distribution over the cathodic area, which is approximately the whole area. In any study of the factors that determine current distribution over an extensive cathode area, the geometry and dimensions of the metal structure and its surrounding medium are determining factors. It is the purpose of this paper to analyze the potential-current relations that control this current distribution, as related to cathodic protection.

\section{Dimensions and Current Distribution}

In cathodic protection, we deal with cells of all shapes and sizes. It has been theoretically demonstrated [2] that the over-all potential-current relations in a large cell may not be truly represented by the results obtained in a model of a very much smaller size. This is true because the potential differences within a cell, through which current is flowing, consist of two kinds of components. One comprises electromotive forces which, for given current densities, are independent of the size of the cell; and the other includes those potential differences resulting from currents flowing through resistances which, for a given resistivity, are determined by dimensions. The smaller cell may therefore not be a true electrical model of the larger one for a given current density unless the resistivity of its electrolyte is so adjusted that the resistive components in the two cells are equal.

If the required adjustment of resistance is impracticable, the potential-current relations found in a small model may not be applicable directly to a large cell. A procedure for determining these relations by direct measurement in the cell therefore seems desirable. This is particularly true in the case of electrical circuits such as those involved in electrolysis mitigation and cathodic protection of underground pipelines where the resistive components may not be realizable on a laboratory scale. Here we have large cathodic areas, and the volume 
of electrolyte is unlimited. In the application of cathodic protection, the position of the anode, through which the external current is supplied, is of considerable practical importance. It is the purpose of this paper to outline a method tried in the laboratory, which might be translated into a field procedure for determining current distribution. Field experience will then determine whether the laboratory procedure is applicable to underground conditions.

Theoretical methods of computing current attenuation along a conductor of great length frequently neglect the role of counter emf and polarization [3], and consider the resistance as the only controlling factor. On the latter assumption, the current density at different points on the cathode varies inversely as the resistance of the current paths from the anode to the respective points, in accordance with Ohm's law. However, any counter emf reduces the current in the same ratio as that of the counter emf to the applied emf at that point. Any increase in counter emf therefore tends to reduce the current at points of higher current density to a greater degree than at points of lower current density. This results in a more uniform current distribution, for a given applied voltage, than if resistance were the sole current-limiting factor. For example, if $i_{1}$ and $i_{2}$ are the currents flowing to unit areas having electrode potentials $e_{1}$ and $e_{2}$, respectively, then [4]

$$
\frac{\partial i_{1}}{\partial i_{2}}=\frac{r_{2}+\frac{\partial e_{2}}{\partial i_{2}}}{r_{1}+\frac{\partial e_{1}}{\partial i_{1}}}
$$

where $r_{1}$ and $r_{2}$ are the resistances of the paths of the currents. For a given metal and environment,

$$
\frac{\partial e_{2}}{\partial i_{2}}=\frac{\partial e_{1}}{\partial i_{1}}=\frac{\partial e}{\partial i}
$$

If $\partial e / \partial i$ becomes very large as compared with the resistances, $r$ and $r_{2}, \partial i_{1} / \partial i_{2}$ approaches 1 . In electroplating, this phenomenon is called "throwing power", which is a function of $\partial e / \partial i$, and the geometry of the cell and may be expressed in several ways. In cathodic protection also, a high value for $\partial e / \partial i$, as compared with resistance, favors throwing power and therefore greater uniformity in current distribution.

In cells of very small dimensions, as in pits and crevices on the metallic surface, the effective resistances $r$ and $r_{2}$ may be small; and the value of $\partial e / \partial i$ probably greater because of larger ionic concentration gradients. These conditions favor a higher throwing power than over large areas free from sharp surface irregularities.

\section{Determination of Current Distribution}

In any study of current distribution, it is essential that a method of determining apparent current density at any point be available. When current distribution is uniform,

$$
i=\frac{I}{A}
$$

where $I$ is the total current; and $A$ is the total area: If $I$ and $A$ can be measured, there is no problem in determining the apparent current density. However, when the current distribution is not uniform, the relation of current density to polarization, if there is such a relation, may be used to determine $i$ at any point where the cathode potential $e_{c}$ can be measured. If the potential $e_{c}$ can be measured without including any resistive components, then the relation, $e_{c}=(f) i$, may be used to determine $i$ at any point in cells, regardless of dimensions.

In order to demonstrate the relation of current distribution to polarization and resistance, a test cell, large enough to obtain a convenient current-density gradient, was used. It consisted of a wooden tank, $3 \mathrm{ft}$ by $10 \mathrm{ft}$ by $1 \mathrm{ft}$ deep, entirely insulated from outside circuits (fig. 1). A $3 / 4$-in. steel tube extended the full length of the tank and a small steel anode, $A$, was in one corner, as indicated. The electrolyte covered the tube by several inches. The problem was to determine the current density at points 1,2 , .: ., 10 inclusive, for a given applied emf, $E_{t}$. Direct measurement of current density at any point was impracticable. However, if the relation of cathode potential $e_{c}$ to $i$ is known, then $i$ can be easily determined. In the present case, the currentdensity gradient along only one dimension is of interest. For this reason current density is expressed as current per unit length of tube. Variation in current density around the tube is disregarded.

In order to obtain the data for a graph showing the relation between $e_{c}$ and $i$, measurement of $e_{c}$ on a given length of tube, on which the apparent current density is assumed to be uniform, is necessary at different values of $I$. The obvious method of obtaining such uniformity is by the use of a parallel anode. This was accomplished by replacing the small steel anode, $A$, with a rod equal to the length of the tank. Then $i=I / l$, where $l$ is the length of tube, uniform current distribution being assumed. For the determination of $e_{c}$, a saturated calomel electrode was placed on the surface of the tube at each numerical location, and the potential difference $\left(e_{c}-E_{s}\right)$, was measured, using the circuit in figure 1 , where $E_{s}$ is the potential of the calomel electrode.

\section{Method of Measurement}

This circuit [5] permitted measurement of the quantities $\left(e_{c}-E_{s}\right)$ and $\left(e_{c}-E_{s}+I r_{s}\right)$, where $r_{s}$ is the resistance between the reference electrode and the cathode surface. For a given current, $I$, the bridge was balanced by adjustment of $X$ until momentary closing of key $K_{1}$, caused no change in the deflection of null-indicator $G$. Since the resistances in arms $D D$ were equal (each 50,000 ohms), then at balance $r_{s}=X$. After balance, the counter emf $V_{g}$ was 
adjusted until there was no deflection of $G$. Then in circuit (1)

$$
\left(e_{c}-E_{s}\right)-V_{g}=\left(I-I_{D}\right) r_{s}-I_{D} D,
$$

and in circuit (2)

$$
V_{g}=\left(I-I_{D}\right) X-I_{D} D .
$$

Subtracting the equation for (2) from that for (1), and since $r_{s}=X$,

$$
\left(e_{c}-E_{s}\right)=2 V_{g l}
$$

where $V_{g l}=$ voltmeter reading.

If key $K_{2}$ be opened and $V_{g}$ be readjusted to a new value $V_{g 2}$ until $G$ again reads zero, then $\left(e_{c}-E_{s}+I r_{s}\right)=V_{g 2}$, where $Y_{g 2}$ is the voltmeter reading. This is equivalent to a potentiometer measurement. When $I r_{s}$ is negligible, use of the bridge is unnecessary, and $\left(e_{c}-E_{s}\right)$ may be observed directly as $V_{g 2}$, for all practical purposes.

The instrumental requirements of this circuit are not stringent. As null indicator $G$, a General Electric galvanometer was satisfactory. In the field, a Weston Model 622 voltmeter with zero-center scale and a resistance of $200,000 \mathrm{ohms} / \mathrm{v}$ may be suitable and more convenient. A calibrated slide-wire rheostat of sufficient current-carrying capacity was suitable for balancing the bridge and reading. This circuit has been used in preliminary field tests but is not yet in suitable portable form.

\section{Potential Criterion of Cathodic Protection}

For each value of $I$, the emf $\left(e_{c}-E_{s}\right)$, was measured at each point in numerical order, and also in the

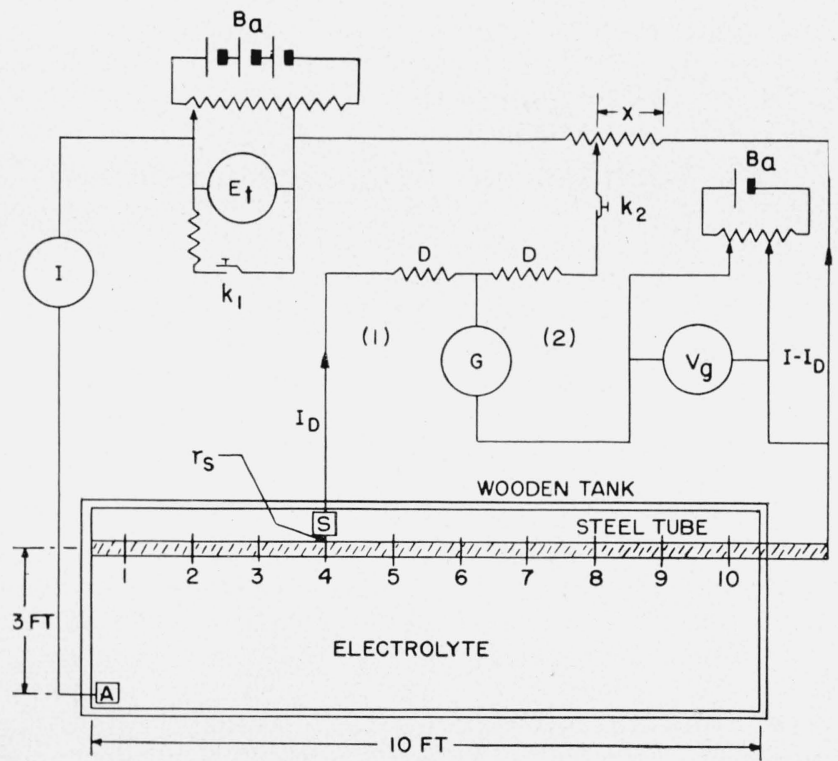

FIgURE 1. Circuit for measuring polarization at points of different current densities along a steel tube. reverse order, making a total of 20 readings. The results are summarized in figure 2 , where two graphs represent the averages, one for tap water and one for 0.1-percent $\mathrm{NaCl}$ solution. The "breaks" occur at potential levels near but slightly below the opencircuit potential of iron in a saturated solution of ferrous hydroxide. At this potential, 0.813 volt, corresponding to a $\mathrm{pH}$ of about 9.5 , the reaction $\mathrm{Fe} \rightleftarrows \mathrm{Fe}^{++}+2 \epsilon$ reaches equilibrium, and corrosion of iron by this process practically ceases [6]. The data in figure 2 were obtained for electrolytes that were undisturbed, except by convection, and were very probably saturated with air. An air-free environment is therefore not a requirement for obtaining the air-free potential by cathodic polarization. The reproducibility of the readings was better than was anticipated for iron in a solution initially having an undefined concentration of ferrous ions.

The protective current, $I_{p}$, indicated in the salt solution was about $2.4 \mathrm{ma} / \mathrm{ft}$ and in the tap water, $1.5 \mathrm{ma} / \mathrm{ft}$. Thus, the corrosiveness in the more conductive salt solution was greater than in the tap water, though not in the ratio of the conductivities, which was approximately ten to one. It is apparent that this large difference in conductivity had no apparent effect on the potential at which the breaks in the polarization curves occurree

Next, the parallel anode was replaced by the point anode $A$, and for a given value of $E_{t}$ and $I, e_{c}$ was observed at the numbered points along the tube. By the use of the graphs in figure 2, the corresponding values of $i$ were then estimated for each point. In table 1 the values of $e_{c}$ and $i$ are given, for each point of observation, in a 0.1 -percent solution of sodium chloride for a current of $12 \mathrm{ma}$. Since the potential of the tube $=e_{0}$, when $i=0$, the difference $\Delta e_{c}=e_{c}-e_{0}$ represents the emf of polarization, also given in table 1.

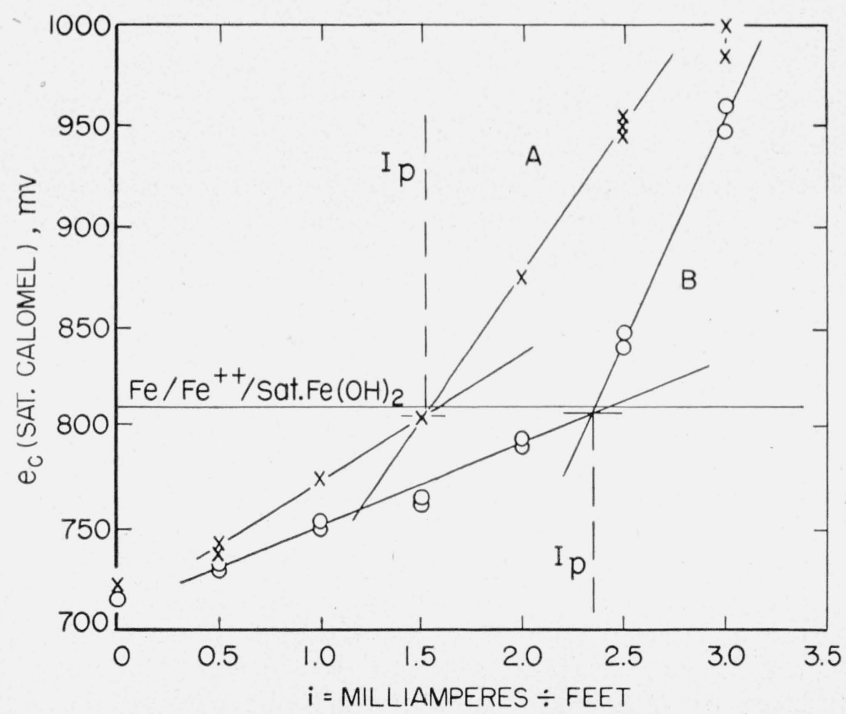

Figure 2. Relation of electrode potential to current density along a steel tube.

A, Tap water; B, 0.1-percent $\mathrm{NaCl}$ solution. 
TABLE 1. Observed potentials and current densities at different points; calculated resistances and current-density components

$e_{o}=715 \mathrm{mv} ; e_{A}=588 \mathrm{mv} ; E_{t}=1,170 \mathrm{mv} ; I=12 \mathrm{ma}$.

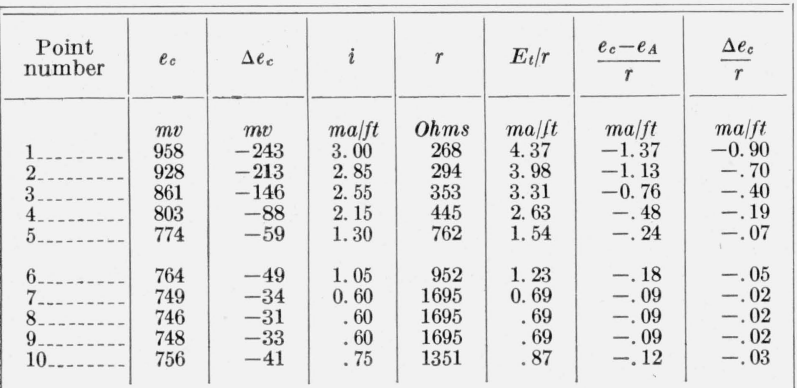

\section{Calculation of Resistance of Current Path}

Let us now analyze the relation of current-density attenuation to the resistance of the current paths from the anode to successive unit lengths of tube. For a given current $I$, the polarized anode potential $=e_{A}$. Then, since the applied $\operatorname{emf}=E_{t}$, and the external resistance was negligible,

$$
\frac{E_{t}-\left(e_{c}-e_{A}\right)}{i}=r,
$$

where $r=$ the resistance of the path of current $i$ from anode $A$ to a given unit length of tube. That is, $r_{1}, r_{2}, \ldots, r_{10}$ are the resistances of the paths of currents $i_{1}, i_{2}, \ldots$., $i_{10}$, respectively. Using eq 2 , the values of $r$ were computed from the corresponding values of $e_{c}$ and $i$, where $E_{t}=1,170 \mathrm{mv}$, and $e_{A}=588 \mathrm{mv}$. These results are also summarized in table 1 .

\section{Polarization}

Rearranging eq 2 , we obtain

$$
i=\frac{E_{t}}{r}-\frac{\left(e_{c}-e_{A}\right)}{r}
$$

where $i$ consists of two components in opposition. One, $E_{t} / r$, with $E_{t}$ constant depends only on $r$; and the other $\left(e_{c}-e_{A}\right) / r$, with $e_{A}$ constant, depends on $r$ and also upon $e_{c}$. The expression $E_{t} / r$ is the primary current distribution representing the current density that would be obtained if there were no counter emf. For example, if the cell were entirely filled uniformly with a conductor of the first class, or if it had identical nonpolarizable electrodes, like copper in copper sulphate solution at low current densities, $\left(e_{c}-e_{A}\right)=0$. Thus $E_{t} / r_{t}$ is the largest current density attainable for a given value of $E_{t}$ and $r$.

If $e_{c}>e_{A}$ and $e_{c}$ is nonpolarizable, having a constant value $e_{0}$, then we have a constant counter emf $\left(e_{0}-e_{A}\right)$, as for example, in a lead-acid storage battery on charge at low current density.

If $e_{c}>e_{A}$ and $e_{c}$ is polarizable, then we have the present case, where $\left(e_{c}-e_{A}\right)$ represents the counter emf in which $e_{c}=e_{0}+\Delta e_{c}$. The amount of polariza-

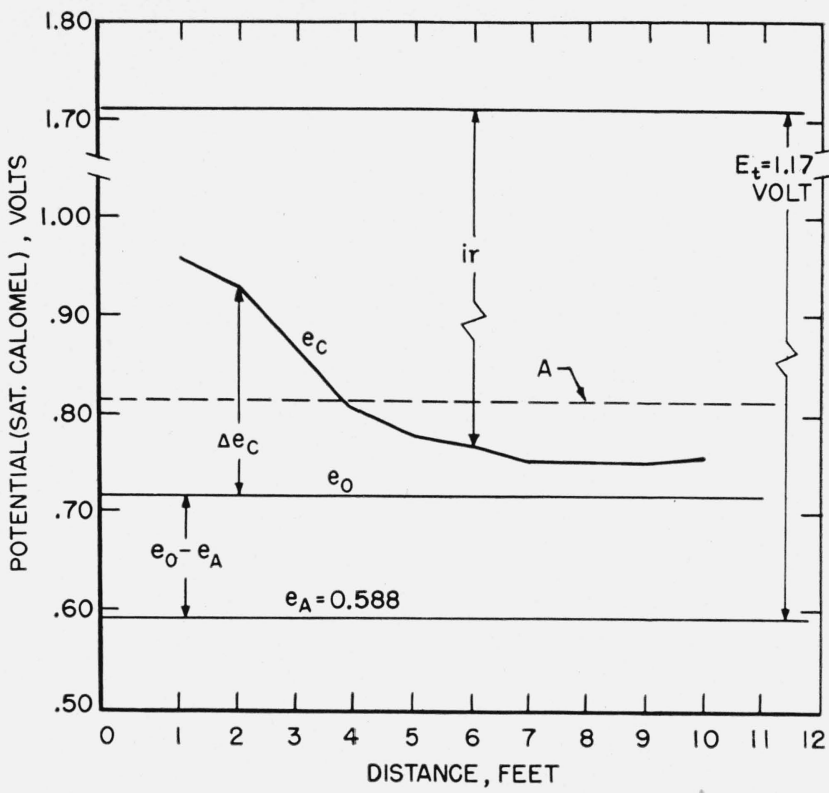

FIGURE 3. Relation between emf and ir components of polarization along a steel tube.

A, Open-circuit potential of iron, 0.813 volt.

tion may be expressed as $\Delta e_{c}$ in millivolts, or as $\Delta e_{c} / r$ in milliamperes. Both indices in table 1 show that the role of polarization in determining currentdensity distribution diminishes at the lower current densities. At the minimum current density, resistance is practically the sole determining factor, as indicated in figure 3 .

The current-distribution curves for salt water (fig. 4) were essentially the same as those obtained in the tap water (fig. 5). For example, cathodic protection defined as that value of $e_{c}$, equal to or above $0.813 \mathrm{v}$, extended approximately to the $4-\mathrm{ft}$ location in each electrolyte; and as shown in (fig. 2), a lower current density was required for protection in the tap water than in the salt solution.

\section{Location of Reference Electrode}

When the tip of the reference electrode was in contact with the steel tube surface, there was a small resistance $r_{s}$ (fig. 1) between the electrode and the surface, which increased with current density (fig. 6). On a copper tube, this resistance was greater by several-fold and may have been due to liberated hydrogen, or to a film resulting from increased alkalinity at the surface.

When the reference electrode was located 18 in. from the tube surface, the observed readings were averages of the true potentials for a range of points extending over a considerable length of the tube. For example, in figure 6 , the potential (800 mv) observed at locations 1 and 2, with the electrode $18 \mathrm{in}$. away was approximately the average potential over a 5-ft length as measured with the reference electrode directly on the surface. The significance of this observation is that, when the potential and 


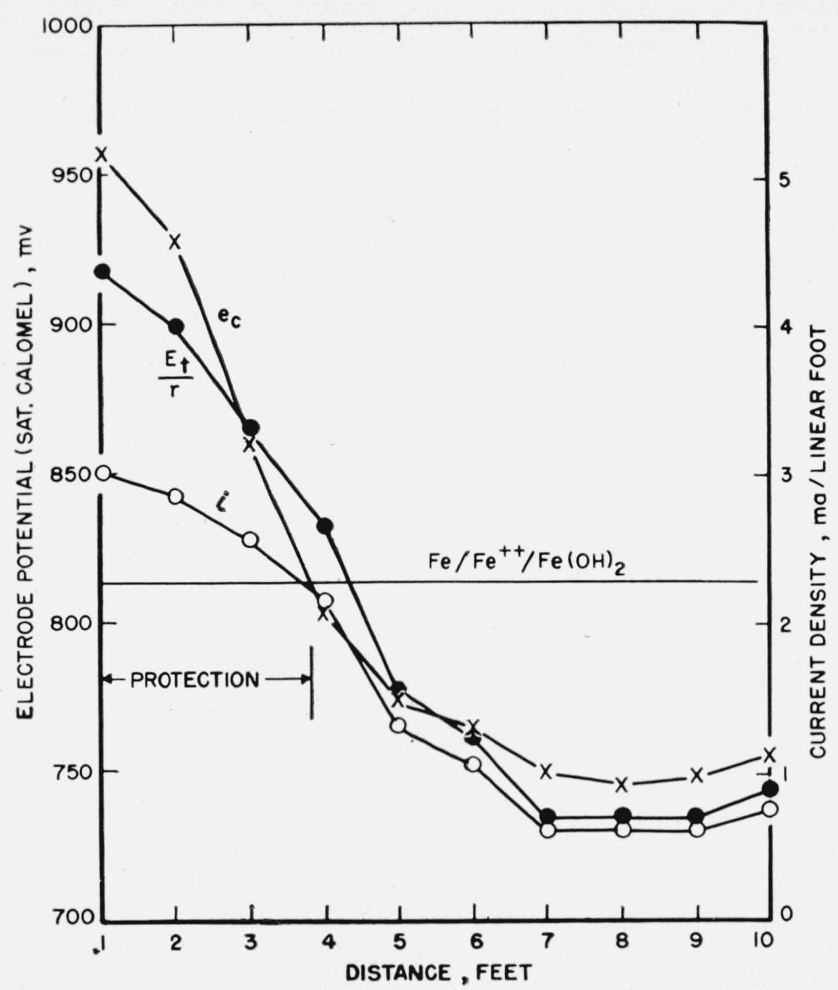

FIGURE 4. Cathodic potential $e_{c}$, current density $i$, and primary current distribution $E_{t} / r$ along a steel tube in $\mathrm{NaCl}$ solution.

0.1-Percent $\mathrm{NaCl}$ solution; $I=12 \mathrm{ma}$

current-density gradients along the tube are steep, the reference electrode must be as near as possible to the surface in order that the observed value approach the magnitude of $e_{c}$ at a given point. At best, the observed value of $e_{c}$ is an average potential over an area that increases with the distance of the reference electrode from the surface. The purpose of reducing this distance to a minimum is not that of reducing the effect of resistance, since this can be balanced out by the bridge measurement, but in order to reduce the observed area to a minimum. Obviously, if the cathode potential is uniform, the requirement of proximity is unnecessary. A more remote location may then be used, the distance from the surface being greater, in proportion to the area of uniformity.

A frequent subterfuge of locating the reference electrode behind the electrode under investigation, or at some other point of minimum current density. may neither eliminate the ir component in the observed potential nor indicate the value of $e_{c}$ at the front surface of the electrode, which is of most importance. The reference electrode should therefore be placed at the exact point, where the value of $e_{c}$ is required, with the least possible disturbance of the lines of current flow. This requires a reference electrode with the smallest exploring tip that is practicable.

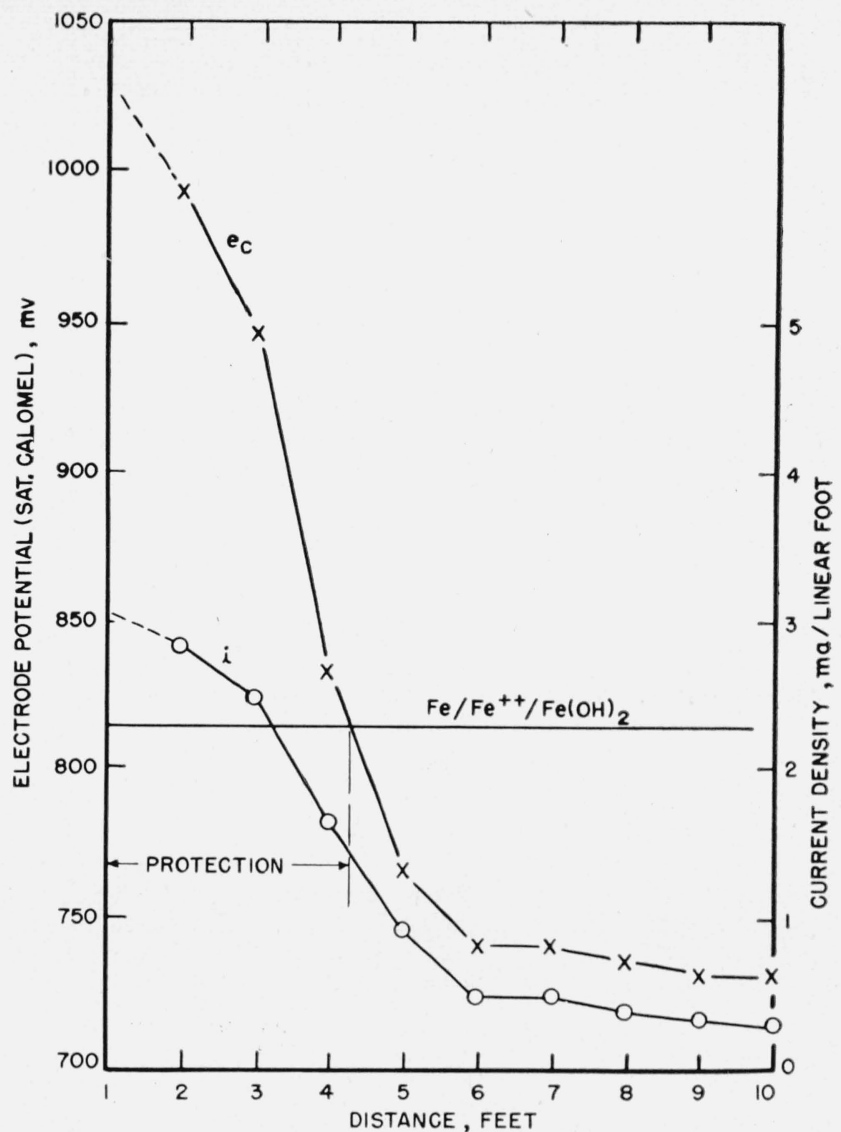

FIGURE 5. Cathodic potential $e_{c}$ and current density $i$ along a steel tube in Washington tap water.

Tap water; $I=12 \mathrm{ma}$.

\section{Suggested Field Procedure for Determin- ing Current Distribution}

In estimating the current distribution over a very extended cathode surface, such as a pipe-line or a very large plane surface, the cathodic polarizationcurrent density relation may be very useful. For determining this relation, a procedure, based on the guard-ring principle [7] in electrical measurements, suggests itself. For example in figure 7, let a parallel three-section line anode be laid as near the pipe surface as possible without distrubing the surface environment. The two end-sections will supply current to unknown lengths of pipe over which the currentdensity varies from a maximum to practically zero at some unknown distance away. By thus eliminating the "end-effect" current from the meter reading, we know the length of pipe which is receiving the measured current $I$, being supplied by the middle section of the anode.

The degree of uniformity of current density within the length $l$, of course, may not be predictable. If the values of $e_{c}$ observed at numerous points within length $l$ and the resistivity of the surrounding medium along the length $l$ are fairly uniform, then assuming 


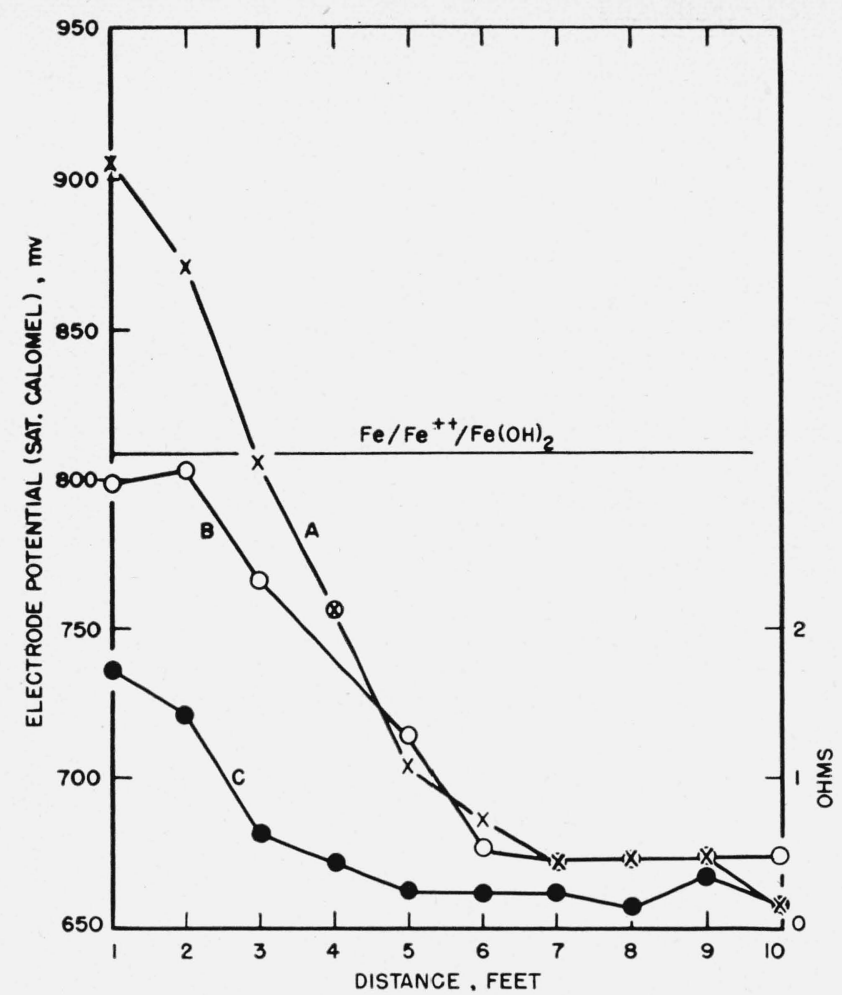

Figure 6. Relation of potential readings and resistance $r_{s}$ along a steel tube, for different positions of the reference electrode.

Steel tube in 0.1-percent $\mathrm{NaCl}$ solution; $12 \mathrm{ma}$; A, reference electrode on surface; $\mathrm{B}$, reference electrode $18 \mathrm{in}$. from surface; $\mathrm{C}$, resistance $r_{s}$ with reference electrode on surface.

that $e_{c}$ is a function of $i$ the current distribution should be uniform. If it is, then a clearly defined potentialcurrent density relation similar to that in figure 2 should be obtainable by the technique outlined in figure 7 . Such a relation may then be used to determine current distribution beyond the ends of length $l$ in a uniform environment.

In the case of a large plane surface, $l$ in Figure 7 may represent the diameter of a disk within a ring having a slightly larger inside diameter, both being in a plane near and parallel to the surface. The measured current, I, will flow to an area approximately equal to that of the disk, the current density $i$ being $4 I / \pi l^{2}$. Then the relation between $e_{c}$ and $i$ may be established within the disk area by the bridge-method used in figure 1 . If $e_{c}=(f) i$, the current-density gradient outside the disk area may be determined by measuring $e_{c}$ at points at increasing distances away. This is very much simpler than the classical but laborious procedure of plotting the equi-potential surfaces around the electrode and then graphically determining the current density lines perpendicular to them.

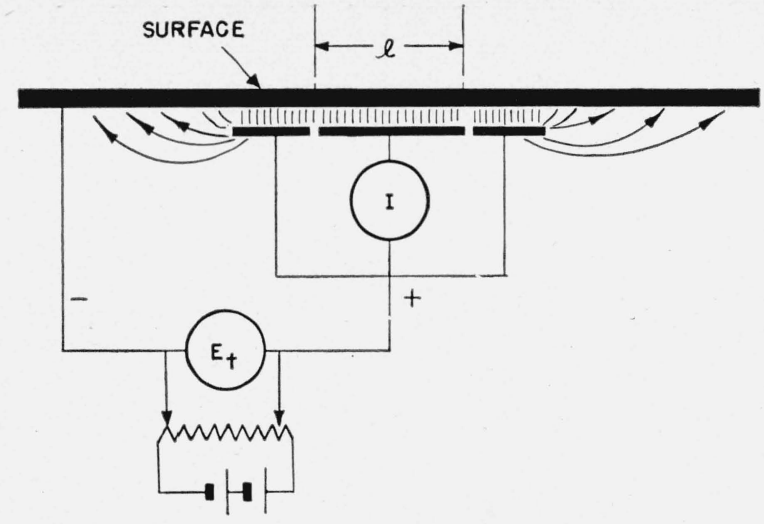

FIGURE 7. Suggested procedure for measuring current flowing to a known length of pipe-line.

\section{$\mathrm{X}$. Conclusions}

Using a reference electrode and a bridge method, which eliminates ir components from the measurement, the relation between current density and the polarized potential of iron was determined in tap water and in a 0.1-percent salt solution. By the application of this relation to a large cathode receiving current from a point anode, the current density at different points on the cathode was obtained from the electrode potentials measured at the same points. The resistances of the current paths from the anode to cathode were computed; and the role of resistance and counter emf in current distribution, thereby demonstrated.

By this procedure, current distribution may be determined, regardless of the dimensions of the cell, if the reference electrode is located very near the cathode surface. If the surface potential is uniform, the reference electrode may be located at some remote point. If the surface potential is not uniform, the reference electrode at a remote point will indicate an average potential over a given area.

When the polarized potential is a known function of current density, the primary current distribution may be computed from the data obtainable by the above procedure. By using the guard-ring principle, the potential-current density relation, and current distribution may be determined over very extensive electrode areas in a uniform environment.

\section{References}

[1] R. B. Mears and R. H. Brown, Trans. Electrochem, Soc. 74, 519 (1938).

[2] J. N. Agar and T. P. Hoar, Discussions on electrode processes, Faraday Soc. No. 1, 158 (1947).

[3] C. Kasper, Trans. Electrochem. Soc. 78, 131 (1940).

[4] H. D. Holler, J. Research NBS 45, 373 (1950) RP2148.

[5] H. D. Holler, J. Electrochem. Soc. 97, 271 (1950).

[6] H. D. Holler, Ind. Eng. Chem. 21, 750 (1929).

[7] J.J. Thomson, Recent researches in electricity and magnetism, p. 233 (Clarendon Press, Oxford, Eng., 1893).

Washington, December 13, 1950. 